\title{
Comparison of visual and refractive results of Toric Implantable Collamer Lens with bioptics for myopic astigmatism
}

\author{
J. F. Alfonso - C. Lisa - L. Fernández-Vega Cueto • \\ P. Fernandes • J. M. González-Méijome • R. Montés \\ Micó
}

Received: 16 April 2012 /Revised: 24 August 2012 / Accepted: 29 August 2012

(C) Springer-Verlag 2012

\begin{abstract}
Purpose To compare visual and refractive results of Toric Implantable Collamer Lens (TICL) and bioptics (ICL plus excimer corneal surgery) to treat myopic astigmatism.

Methods Eighty-one eyes underwent TICL implantation and 83 eyes were treated with bioptics (corneal ablation was performed between 1.5 and 6 months after ICL implantation). Uncorrected distance visual acuity (UDVA), corrected distance visual acuity (CDVA), refraction, adverse events, safety, and efficacy were evaluated 12 months postoperatively.

Results At 12 months postoperatively, the mean spherical equivalent was $-0.15 \pm 0.36$ diopters (D) in the TICL group and $-0.08 \pm 0.26 \mathrm{D}$ in the bioptics group $(p=0.099)$. Sixtysix $(81.5 \%)$ and $78(94.0 \%)$ eyes were within $\pm 0.50 \mathrm{D}$ for TICL and bioptics groups, respectively. The mean Snellen UDVA was not statistically different between both procedures $(p=0.909) ; 53(65.4 \%)$ and $54(65.1 \%)$ eyes
\end{abstract}

J. F. Alfonso $(\varangle) \cdot$ C. Lisa $\cdot$ L. Fernández-Vega Cueto

Fernández-Vega Ophthalmological Institute,

Avda. Dres. Fernández-Vega 114,

33012 Oviedo, Spain

e-mail: j.alfonso@fernandez-vega.com

\section{J. F. Alfonso}

Surgery Department, School of Medicine, University of Oviedo, Asturias, Spain

\section{P. Fernandes · J. M. González-Méijome}

Clinical \& Experimental Optometry Research Lab;

Center of Physics, School of Sciences, University of Minho,

Braga, Portugal

R. Montés Micó

Optics Department, Faculty of Physics, University of Valencia,

Valencia, Spain achieved at least $20 / 25$ or better in TICL and bioptics groups, respectively. No eye had lost more than two lines of CDVA, and $32.1 \%$ of eyes (26/81) in the TICL group and $57.8 \%$ of eyes $(48 / 83)$ in the bioptics group had better postoperative UDVA than preoperative CDVA $(p<0.001)$. Safety was not statistically different between groups $(p=$ 0.464 ) while efficacy was significantly higher in the bioptics group $(p=0.000)$. Two eyes with a TICL were treated to correct TICL decentration.

Conclusions Bioptics showed slightly better outcomes in some clinical measures such as uncorrected visual acuity, efficacy, and refractive predictability. TICL implantation shows reliable results similar to bioptics. A single procedure with TICL implantation might be preferred, eliminating the inherent risks of laser treatments and the risks of a second surgical procedure.

Keywords Implantable Collamer Lens · Bioptics · Excimer laser

\section{Introduction}

Keratorefractive surgeries, such as photorefractive keratectomy (PRK) and LASIK, have successfully been performed to treat myopic astigmatism. Although the outcomes of the procedure were excellent for treating mild to moderate myopic astigmatism, such results do not appear to be as reproducible when used to correct higher levels of myopia and astigmatism [1-3].

The Implantable Collamer Lens (Visian ICL; STAAR Surgical, Nidau, Switzerland) is a foldable posterior phakic intraocular lens (pIOL) that can be used to correct high myopia up to -18.00 diopters (D) and provides stable visual 
outcomes [4-7]. Before the advent of the Toric Implantable Collamer Lens (TICL, STAAR Surgical), the Visian ICL could only correct the spherical component of the refractive error and, as a result, coexisting refractive astigmatism had to be treated by either keratorefractive procedures such as incisional keratotomy or excimer laser ablation. Combining surgical procedures was initially described by Zaldivar et al. [8], who termed bioptics to the use of LASIK after pIOL implantation to treat extreme myopia and myopia combined with astigmatism. Some studies reported that bioptics is a valuable option for treating residual refractive errors [9-12] with an improved predictability and similar safety when compared with single pIOL surgery.

Currently, toric ICL implants have demonstrated to be safe and effective in correcting myopia with astigmatism, with excellent visual and refractive results [13-16]. However, there is only one study that directly compares the clinical results between TICL implantation and bioptics [17]. The purpose of this study was to evaluate the clinical results and compare the safety, efficacy, and predictability between TICL implantation and bioptics to treat eyes with moderate to high myopia with astigmatism.

\section{Patients and methods}

The medical records of 164 eyes of 106 patients who underwent implantation of a Collamer pIOL to correct myopia and astigmatism at the Fernández-Vega Ophthalmological Institute (Oviedo, Spain) were evaluated retrospectively. Eighty-one eyes of 53 patients underwent TICL implantation from September 2004 to April 2009 and 83 eyes of 53 patients had bioptics from June 2003 to October 2008. In the bioptics group, data were collected from patients who had previously undergone spherical Visian ICL (model V4) implantation and subsequent residual refractive errors treated by excimer laser between 1.5 and 6 months after ICL implantation.

At the time of the surgery, all patients were fully informed of the details and possible risks of the surgical procedures. Written informed consent was obtained from all patients before surgery in accordance with the Declaration of Helsinki and the study was approved by an institutional review board.

Exclusion criteria included clinical signs of intraocular inflammation, endothelial dystrophy, cataract, glaucoma, macular degeneration or retinopathy, progressive myopia, previous intraocular surgery, age $<22$ years, anterior chamber depth $<2.8 \mathrm{~mm}$, and endothelial cell density (ECD) $<2,000$ cell $/ \mathrm{mm}^{2}$. Before the ICL implantation, patients had a complete ophthalmologic examination, including manifest and cycloplegic refraction, keratometry, corneal topography, and pachymetry using the Orbscan II (Bausch \& Lomb, Rochester, NY, USA), ECD (SP 3000P; Topcon Europe Medical, The Netherlands), slit-lamp examination, Goldmann applanation tonometry, and binocular indirect ophthalmoscopy through dilated pupils.

\section{ICL size and power calculation}

All eyes were implanted with the latest ICL models (ICLV4 for myopia and TICL for myopia with astigmatism). The ICL size was individually determined based on the horizontal white-to-white distance (WTW) and anterior chamber depth (ACD) measured with Orbscan II following the manufacturer's recommendations. The size was calculated by adding $0.5 \mathrm{~mm}$ to the horizontal WTW measurement. Power calculation for both ICL was performed using the software provided by the manufacturer using a modified vertex formula. The ICL and TICL surgical procedure was the same as reported previously by the authors [18-20]. In all eyes, at least 1 week before ICL implantation, two peripheral laser Nd:YAG iridotomies were performed . All ICL and TICL implantations were performed through a 3.2$\mathrm{mm}$ clear corneal tunnel incision in the horizontal meridian using peribulbar anesthesia. Cycloplegic and phenylephrine eye drops were instilled $30 \mathrm{~min}$ before surgery and povidone-iodine $5 \%, 5 \mathrm{~min}$ before surgery. The anterior chamber was filled with sodium hyaluronate $1 \%$ (Provisc), which was completely removed at the end of surgery. The ICL and

Table 1 Preoperative demographics of eyes that underwent Toric Implantable Collamer Lens implantation or bioptics for the correction of myopic astigmatism

\begin{tabular}{|c|c|c|c|}
\hline & TICL & Bioptics & $p$ value \\
\hline No. of eyes (patients) & $81(53)$ & $83(53)$ & 0.054 \\
\hline M/F (eyes) & $22 / 31$ & $16 / 37$ & 0.002 \\
\hline Mean age (range) (years) & $32.8 \pm 6.5(22$ to 46$)$ & $30.5 \pm 5.4(22$ to 45$)$ & 0.024 \\
\hline Refractive sphere (D) ${ }^{\mathrm{a}}$ & $-5.07 \pm 2.99(-11.00$ to 0.50$)$ & $-9.34 \pm 3.23(-14.00$ to -2.50$)$ & 0.000 \\
\hline Refractive cylinder (D) ${ }^{\mathrm{a}}$ & $-3.09 \pm 1.18(-6.00$ to -0.50$)$ & $-2.55 \pm 1.06(-6.00$ to -0.50$)$ & 0.002 \\
\hline
\end{tabular}

TICL Toric Implantable Collamer Lens

${ }^{a}$ Values represented as mean and standard deviation 


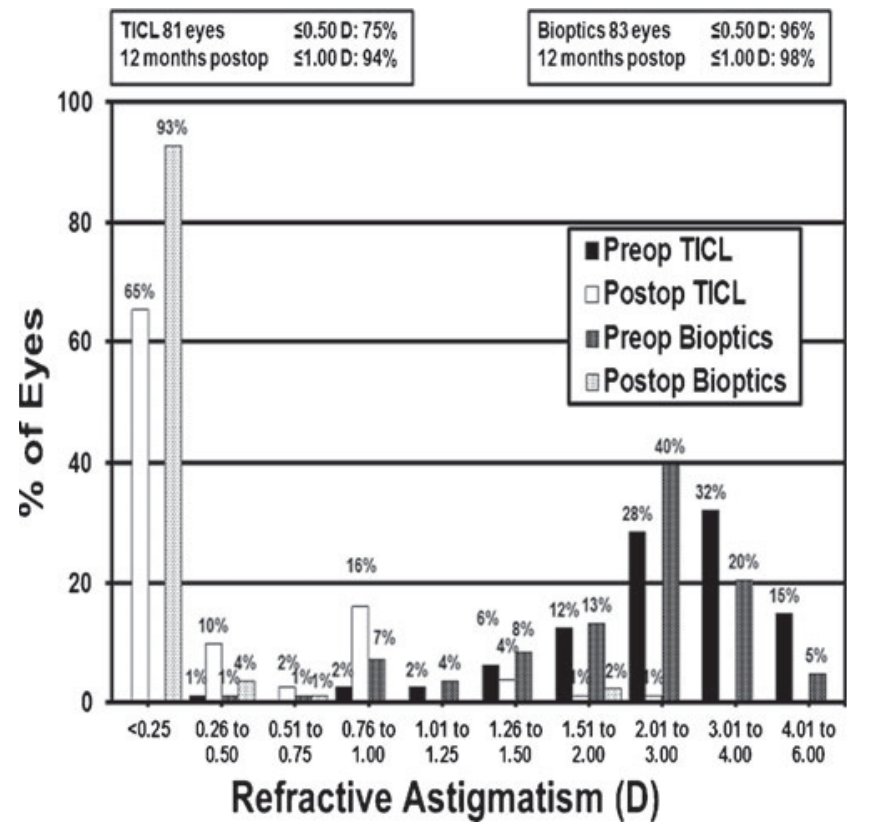

Fig. 1 Preoperative (preop) versus 12-month postoperative refractive cylinder in diopters (D) after TICL implantation and bioptics

TICL were introduced into the anterior chamber with an injector cartridge designed by STAAR Surgical. In TICL implantation, to control for potential cyclotorsion when the patient is in a supine position, the surgeon marked the zero horizontal axis at the 3- and 9-o'clock limbus using a marking pen with the patient sat upright at a slit lamp. The surgeon also used a Mendez ring to measure the required rotation from horizontal during the surgical procedure and the lens was rotated to the required axis using a modified intraocular spatula. Tobramycin and dexamethasone $0.1 \%$ (Tobradex, Alcon Laboratories, Inc.) eye drops were used four times a day for 7 days, after which diclofenac sodium eye drops (Voltaren; Novartis Pharmaceuticals, Basel, Switzerland) were started four times a day for 2 weeks. In cases of bilateral implantation, the second eye was operated within the first week after the fellow eye surgery.
Laser surgery

LASIK or PRK were performed at least 1.5 months after ICL surgery and all the eyes showed a stable refraction and corneal topographic pattern for at least 1.5 months before performing LASIK or PRK. Both surgeries were done by the same surgeon (JFA). Laser surgical techniques followed common procedures using the IntraLase FS femtosecond laser and Visx Star S4 (Abbott Medical Optics, Inc.) excimer laser system. In PRK, the epithelial sheet was partially removed from the Bowman layer after the application of $20 \%$ alcohol for $30 \mathrm{~s}$ and laser ablation was applied. LASIK was performed in 70 eyes and PRK in 13 eyes depending on the corneal thickness and ablation depth of each patient. All surgical procedures were uneventful and without post-surgical complications within the follow-up time presented in this study.

\section{Postoperative assessment}

Both after ICL and after laser surgery all the patients fulfilled the follow-up protocol in which the examination visits were carried out at 1 day, 1 week, and 1 month, and then every 3 months as necessary. Data obtained in each postoperative follow-up visit included uncorrected distance visual acuity (UDVA), corrected distance visual acuity (CDVA), slit-lamp examination, refraction, ECD, fundus examination, intra-ocular pressure (IOP), and central separation between the lens anterior surface and the posterior surface of the ICL (Vault) were performed. For averaging, visual acuities were converted to logMAR values; then, the means and standard deviations were back-calculated to Snellen acuity. Sphero-cylindrical refractive results were converted into vectors expressed by three dioptric powers: $\mathrm{M}, \mathrm{J}_{0}$, and $\mathrm{J}_{45}$; with $\mathrm{M}$ being equal to the spherical equivalent (SE) of the given refractive error, and $\mathrm{J}_{0}$ and $\mathrm{J}_{45}$ the two Jackson crossed cylinders equivalent to the conventional cylinder. Manifest refractions in conventional script notation ( $\mathrm{S}$ [sphere], $\mathrm{C}$ [cylinder], $\alpha$ [axis]) were converted to power vector coordinates and overall blurring strength using the formulas described by

Table 2 Mean values of vectorial decomposition components before and after TICL implantation and bioptics

\begin{tabular}{|c|c|c|c|c|c|c|}
\hline & \multicolumn{3}{|l|}{ Preoperative } & \multicolumn{3}{|c|}{12 months postoperative } \\
\hline & $\begin{array}{l}\text { TICL } \\
\text { Mean } \pm \text { SD }\end{array}$ & $\begin{array}{l}\text { Bioptics } \\
\text { Mean } \pm \text { SD }\end{array}$ & $p$ value & $\begin{array}{l}\text { TICL } \\
\text { Mean } \pm \text { SD }\end{array}$ & $\begin{array}{l}\text { Bioptics } \\
\text { Mean } \pm \text { SD }\end{array}$ & $p$ value \\
\hline M & $-6.62 \pm 2.84$ & $-10.61 \pm 3.27$ & 0.000 & $-0.15 \pm 0.36$ & $-0.08 \pm 0.26$ & 0.099 \\
\hline $\mathrm{J}_{0}$ & $0.87 \pm 1.16$ & $0.89 \pm 0.80$ & 0.410 & $0.09 \pm 0.22$ & $0.02 \pm 0.12$ & 0.030 \\
\hline $\mathrm{J}_{45}$ & $0.02 \pm 0.81$ & $0.02 \pm 0.69$ & 0.984 & $-0.01 \pm 0.16$ & $0.01 \pm 0.11$ & 0.911 \\
\hline
\end{tabular}

$B O L D$ the difference between groups is statistically significant

TICL Toric Implantable Collamer Lens; J0 Jackson cross-cylinder, axes at $180^{\circ}$ and $90^{\circ} ; \mathrm{J} 45$ Jackson cross-cylinder, axes at $45^{\circ}$ and $135^{\circ} ; M$ spherical equivalent 
Thibos and Horner [21]: $\mathrm{M}=\mathrm{S}+\mathrm{C} / 2 ; \mathrm{J}_{0}=(-\mathrm{C} / 2)^{*} \cos (2 \alpha)$; $\mathrm{J}_{45}=(-\mathrm{C} / 2)^{*} \sin (2 \alpha)$ and $\mathrm{B}=\left(\mathrm{M}^{2}+\mathrm{J}_{0}{ }^{2}+\mathrm{J}_{45}{ }^{2}\right)^{1 / 2}$.

Data analysis was performed using SPSS for Windows version 16.01 (SPSS Inc. Chicago, IL, USA). Normality of data was checked by Kolmogorov-Smirnov test and analyzed using the Wilcoxon rank-sum test and Mann-Whitney $U$ test to explore statistical differences for refractive and visual acuity scores among different groups (TICL vs. bioptics). Bivariate correlations between attempted versus achieved refraction was analyzed using non-parametric (Spearman's coefficient) correlation analysis. Differences were considered to be statistically significant when the $p$ value was $<0.05$.

\section{Results}

The mean age of the 53 patients of each group was 32.8 years \pm 6.5 (SD) (range 22 to 46 years) and $30.5 \pm 5.4$ in the TICL and bioptics group, respectively. In the bioptics group, corneal ablations were performed at $4.13 \pm$ 1.17 months (range: 1.5 to 6 months) after ICL implantation by LASIK in 70 eyes and PRK in 13 eyes. Mean follow-up was $12.9 \pm 5.4$ months (range: 6 to 23 months) in the TICL group and $12.7 \pm 4.2$ months (range: 5 to 20 months) in the bioptics group. Preoperative descriptive statistics including age and refraction for both groups is shown in Table 1. In the bioptics group, mean $\mathrm{SE}$ and refractive cylinder after ICL implantation and prior to excimer laser treatment were $-0.56 \pm 0.63 \mathrm{D}$ (range: -2.00 to $1.00 \mathrm{D}$ ) and $-1.26 \pm 0.86 \mathrm{D}$ (range: -3.75 to $0.00 \mathrm{D}$ ), respectively.

\section{Refractive outcomes}

Figure 1 presents the distribution of the manifest refractive cylinder before both surgeries compared with the 12-month visit outcomes. There were no significant differences in outcomes between eyes treated with LASIK and eyes treated with PRK. At the 12-month follow-up visit, 76 (93.8\%) and $81(97.6 \%)$ of eyes had $\leq 1.0 \mathrm{D}$ of cylinder for TICL and bioptics groups, respectively. Furthermore, only one eye in each group had preoperative refractive cylinder of $0.50 \mathrm{D}$, whereas $61(75.3 \%)$ and $80(96.4 \%)$ had $\leq 0.5 \mathrm{D}$ of postoperative refractive cylinder for TICL and bioptics groups, respectively.

The distribution of the refractive components after vector conversion, before and after the different surgical procedures, is shown in Table 2. Despite that the mean preoperative refractive cylinder was statistically different between groups, there were no statistically significant differences in $\mathrm{J} 0$ and $\mathrm{J} 45$ astigmatic components. At 12 months postoperatively, mean preoperative manifest SE improved from $-6.62 \pm 2.83 \mathrm{D}$ to $-0.15 \pm 0.36 \mathrm{D}$ in the TICL group and from $-10.61 \pm 3.27 \mathrm{D}$ to $-0.08 \pm 0.26 \mathrm{D}$ in the bioptics group. The difference was not statistically significant for M (SE) and $\mathrm{J} 45$ component ( $p=0.099$ and $p=0.911$, respectively).

Figure $2 \mathrm{a}$ and $\mathrm{b}$ shows the 12-month results for the astigmatic components of the power vector as represented by the two-dimensional vector plot $\left(\mathrm{J}_{0}, \mathrm{~J}_{45}\right)$. It is visible the spread of data preoperatively and the concentration of data around the origin $(0,0$ coordinates $)$ after both procedures.

a

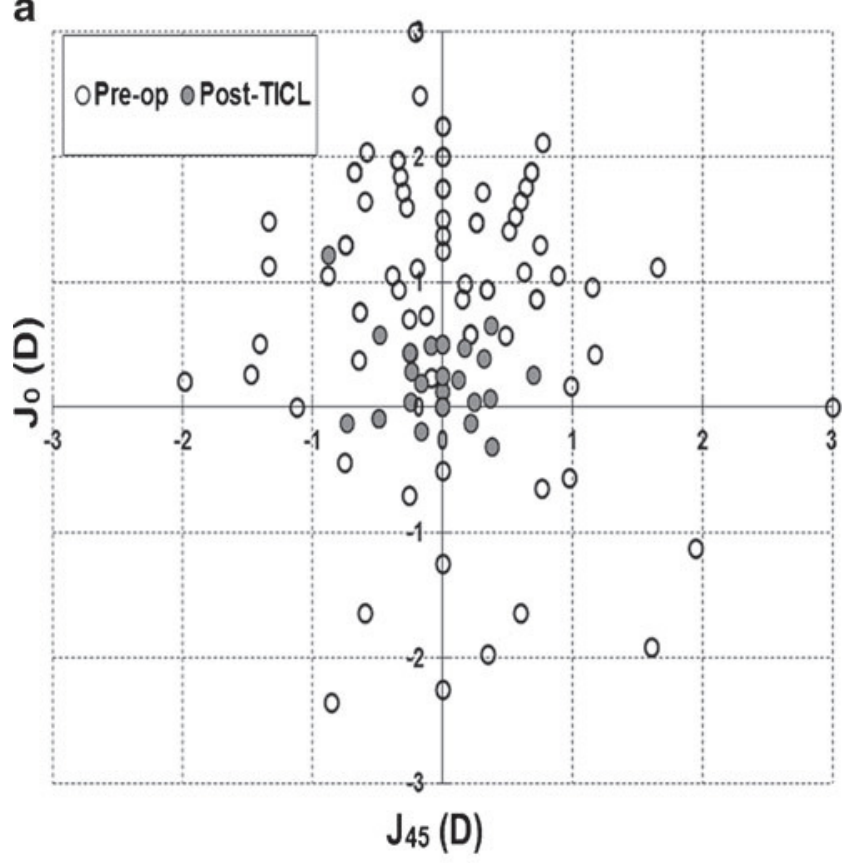

b

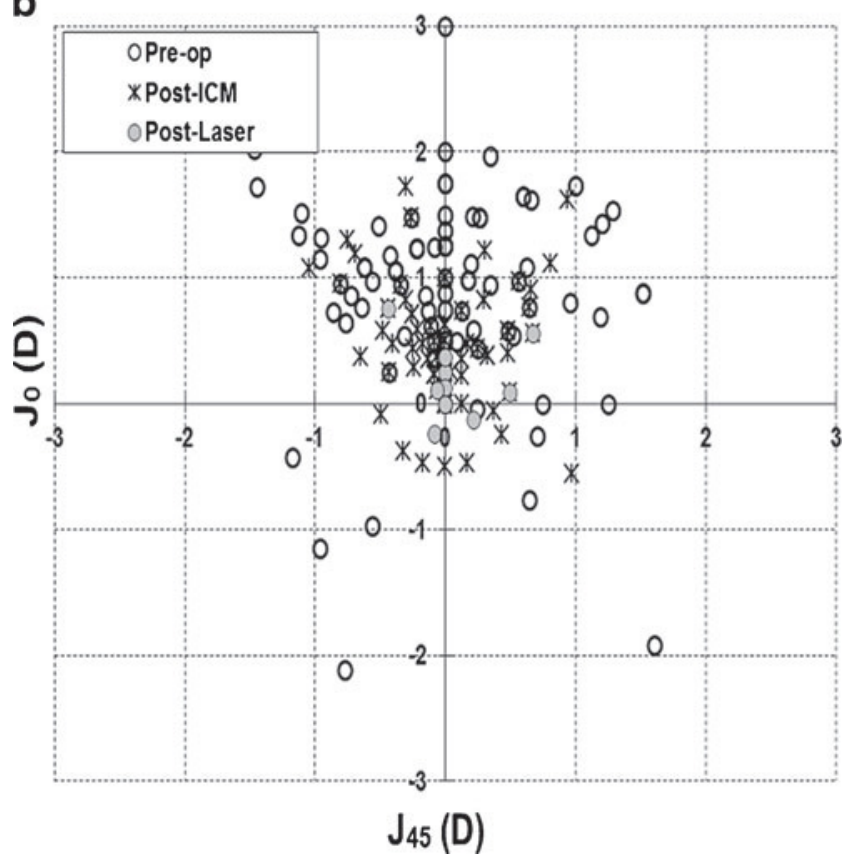

Fig. 2 Scatter plot of the astigmatic vectors $\left(\mathrm{J}_{0}\right.$ and $\left.\mathrm{J}_{45}\right)$ before and after TICL (a) and bioptics (b) treatment. The more central location of postoperative data represents the reduction of preoperative astigmatism 
Distribution of SE after both surgical procedures is shown in Fig. 3. The percentage of eyes with SE within $0.50 \mathrm{D}$ and 1.00 $\mathrm{D}$ was higher in the bioptics group than in the TICL group and all eyes of both groups had a SE lower than 2.0 D. Sixty-six $(81.5 \%)$ and $78(94.0 \%)$ eyes were within $\pm 0.50 \mathrm{D}$ for SE component for TICL and bioptics groups, respectively; 79 $(97.5 \%)$ and $82(98.8 \%)$ eyes were within $\pm 1.00 \mathrm{D}$ of the desired refraction $\left(r^{2}=0.98\right.$ and $r^{2}=0.99$ for attempted vs. achieved correlation analysis, for TICL and bioptics group, respectively). For $\mathrm{J}_{0}$ astigmatic component, 78 (96.3\%) and $81(97.6 \%)$ eyes were within $\pm 1.00 \mathrm{D}\left(r^{2}=0.96\right.$ and $r^{2}=0.98$ for TCIL and bioptics, respectively) while for the $\mathrm{J}_{45}$ component $78(96.3 \%)$ and $82(98.8 \%)$ eyes were within $\pm 1.00 \mathrm{D}$ ( $r^{2}=0.94$ and $r^{2}=0.98$ for TCIL and bioptics, respectively), as shown in Fig. 4a, b and c, respectively.

\section{Visual outcomes}

Table 3 presents pre-and postoperative visual outcomes. The results were similar or better in the TICL group than those of the bioptics group in terms of visual acuity. At 12 months, no statistical difference was noted in mean UDVA between the two groups $(0.81 \pm 0.21$ vs. $0.81 \pm 0.17$ for TICL and bioptics respectively, $p=0.909)$, however, mean CDVA was significantly better in the TICL group $(0.90 \pm 0.14$ vs. $0.84 \pm 0.15$ for TICL and bioptics, respectively, $p=.016$ ). Efficacy index (mean postoperative UDVA/mean preoperative CDVA) was significantly higher in bioptics $(p<.001)$, while the differences in safety index (mean postoperative CDVA/mean preoperative CDVA) were not statistically significant between the two groups $(p=0.464)$.

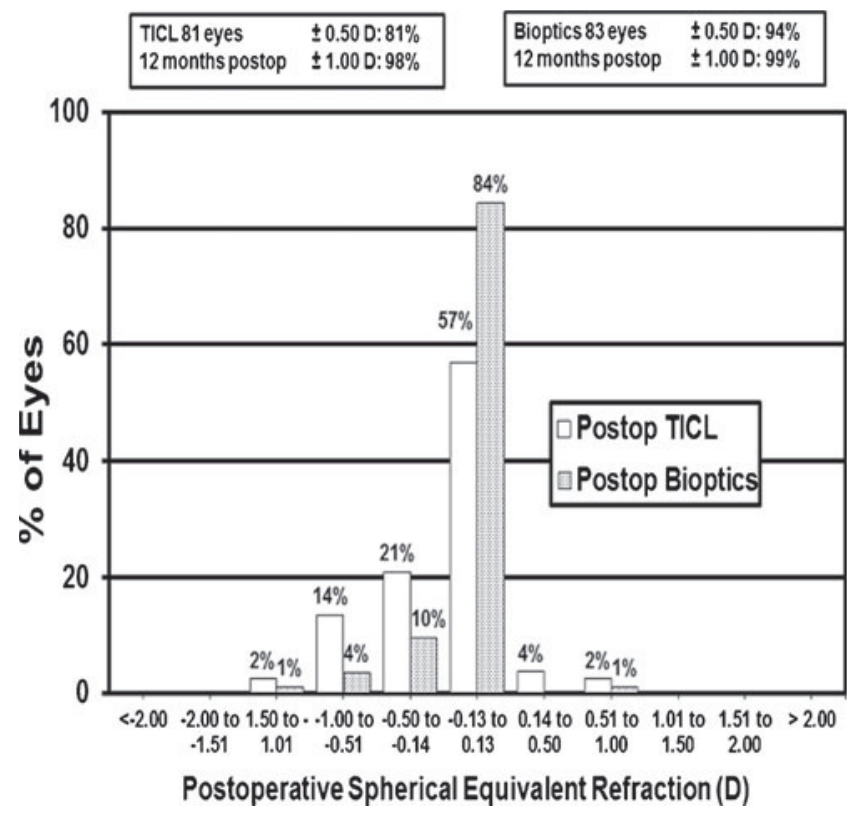

Fig. 3 Twelve month postoperative distribution of spherical equivalent (SE) in diopters (D) for TICL implantation and bioptics
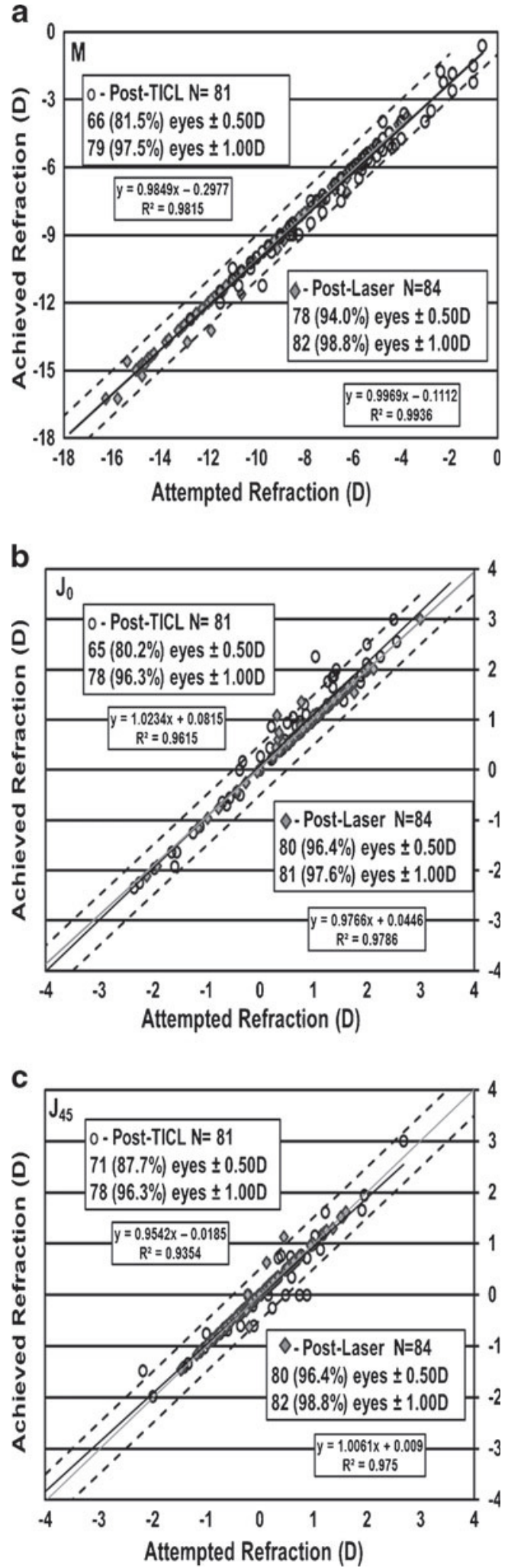

Fig. 4 Plots of achieved correction against attempted correction (predictability) as spherical equivalent (M) (a) and the astigmatic components $\mathrm{J}_{0}$ (b) and $\mathrm{J}_{45}$ (c) in diopters (d) in TICL and bioptics groups 
Table 3 Pre- and postoperative visual outcomes in eyes that underwent Toric Implantable Collamer Lens implantation $(n=81)$ or bioptics $(n=83)$ for the correction of myopic astigmatism

\begin{tabular}{|c|c|c|c|c|c|c|}
\hline & \multicolumn{3}{|l|}{ UDVA } & \multicolumn{3}{|l|}{ CDVA } \\
\hline & $\begin{array}{l}\text { TICL } \\
\text { Mean } \pm \text { SD }\end{array}$ & $\begin{array}{l}\text { Bioptics } \\
\text { Mean } \pm \text { SD }\end{array}$ & $p$ value & $\begin{array}{l}\text { TICL } \\
\text { Mean } \pm \text { SD }\end{array}$ & $\begin{array}{l}\text { Bioptics } \\
\text { Mean } \pm \text { SD }\end{array}$ & $p$ value \\
\hline Preoperative & - & - & - & $\begin{array}{l}0.82 \pm 0.17 \\
(0.4 \text { to } 1.0)\end{array}$ & $\begin{array}{l}0.75 \pm 0.19 \\
(0.2 \text { to } 1.0)\end{array}$ & 0.012 \\
\hline Postoperative & $\begin{array}{l}0.81 \pm 0.21 \\
(0.4 \text { to } 1.0)\end{array}$ & $\begin{array}{l}0.81 \pm 0.17 \\
(0.4 \text { to } 1.0)\end{array}$ & 0.909 & $\begin{array}{l}0.90 \pm 0.14 \\
(0.4 \text { to } 1.0)\end{array}$ & $\begin{array}{l}0.84 \pm 0.15 \\
(0.5 \text { to } 1.0)\end{array}$ & 0.016 \\
\hline Safety index & & & & $\begin{array}{l}1.12 \pm 0.17 \\
(0.75 \text { to } 1.75)\end{array}$ & $\begin{array}{l}1.14 \pm 0.18 \\
(0.75 \text { to } 1.75)\end{array}$ & 0.464 \\
\hline Efficacy index & $\begin{array}{l}0.98 \pm 0.20 \\
(0.50 \text { to } 1.75)\end{array}$ & $\begin{array}{l}1.09 \pm 0.19 \\
(0.50 \text { to } 1.75)\end{array}$ & 0.000 & & & \\
\hline
\end{tabular}

Values represented as mean \pm standard deviation (range)

TICL Toric Implantable Collamer Lens, $C D V A$ corrected distance visual acuity, UDVA uncorrected distance visual acuity

Mean postoperative UDVA was $20 / 63$ or better in all eyes in either groups, with $53(65.4 \%)$ and $54(65.1 \%)$ eyes achieving at least 20/25 or better in TICL group (Fig. 5) and bioptics group (Fig. 6), respectively $(p=0.0909)$. Mean postoperative CDVA was 20/40 or better in all eyes in either groups, with $44(54.3 \%)$ and $27(32.5 \%)$ eyes achieving at least $20 / 20$ or better in TICL and bioptics group, respectively $(p<0.001)$.

Changes in CDVA (safety) after both procedures are shown in Fig. 7. In the TICL group, no eye had lost more than two lines of CDVA, 1 (1.2\%); 2 (2.5\%) eyes lost two and one lines while in the bioptics group $2(2.4 \%)$ and four $(4.8 \%)$ eyes lost two lines and one line, respectively. Seventy-eight

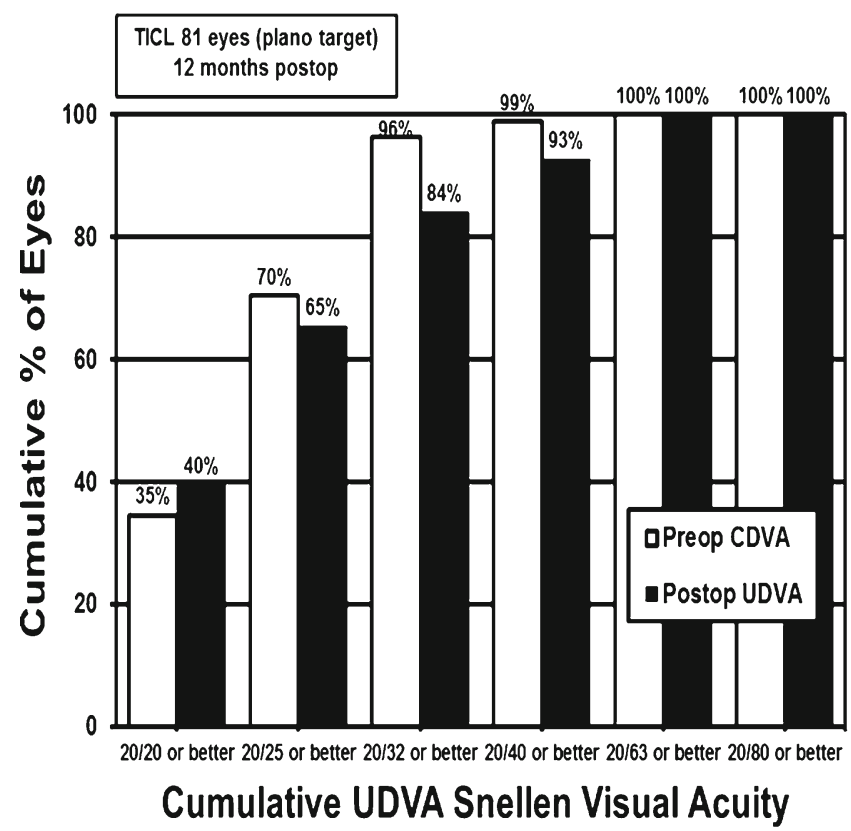

Fig. 5 Preoperative cumulative CDVA Snellen acuity versus postoperative UDVA after TICL implantation eyes $(96.3 \%)$ in TICL and 77 eyes $(92.8 \%)$ in bioptics achieved improved CDVA compared with CDVA at baseline, $(p=0.414)$, and $32.1 \%$ of eyes $(26 / 81)$ in the TICL and $57.8 \%$ of eyes $(48 / 83)$ in the bioptics group gained better postoperative UDVA than preoperative CDVA $(p<0.001)$.

\section{Adverse events}

There were no intraoperative complications. There were no cases of pupillary block or anterior subcapsular cataract during the follow-up period of the study. In the bioptics group, no dehiscence was observed in the ICL incision or dislocation or decentration of the ICL due to laser treatment.

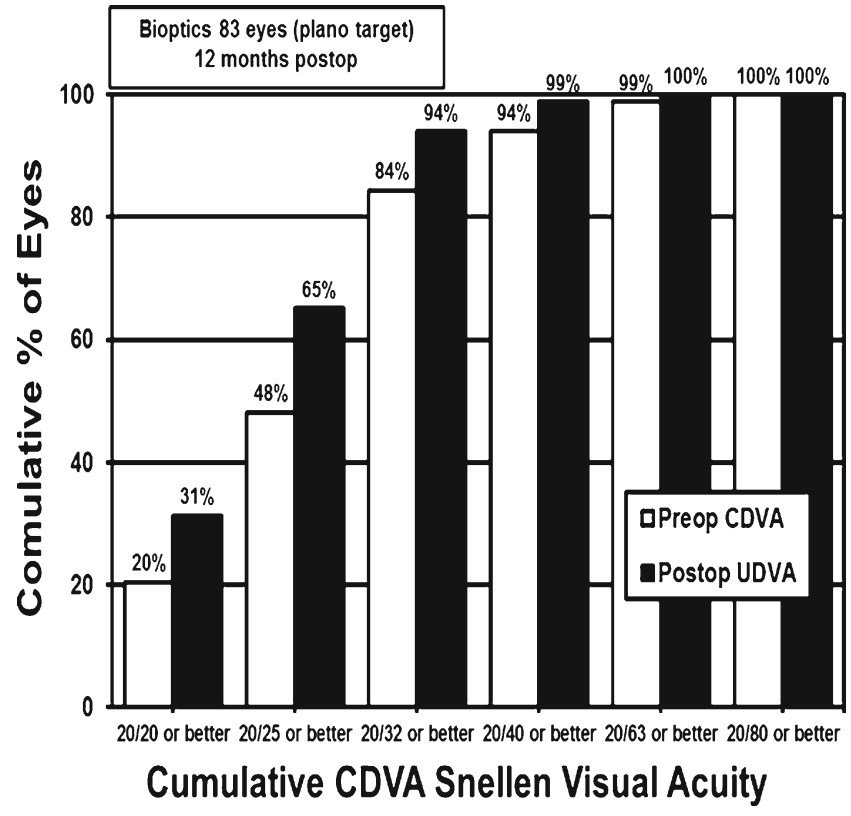

Fig. 6 Preoperative cumulative CDVA Snellen acuity versus postoperative UDVA after bioptics 


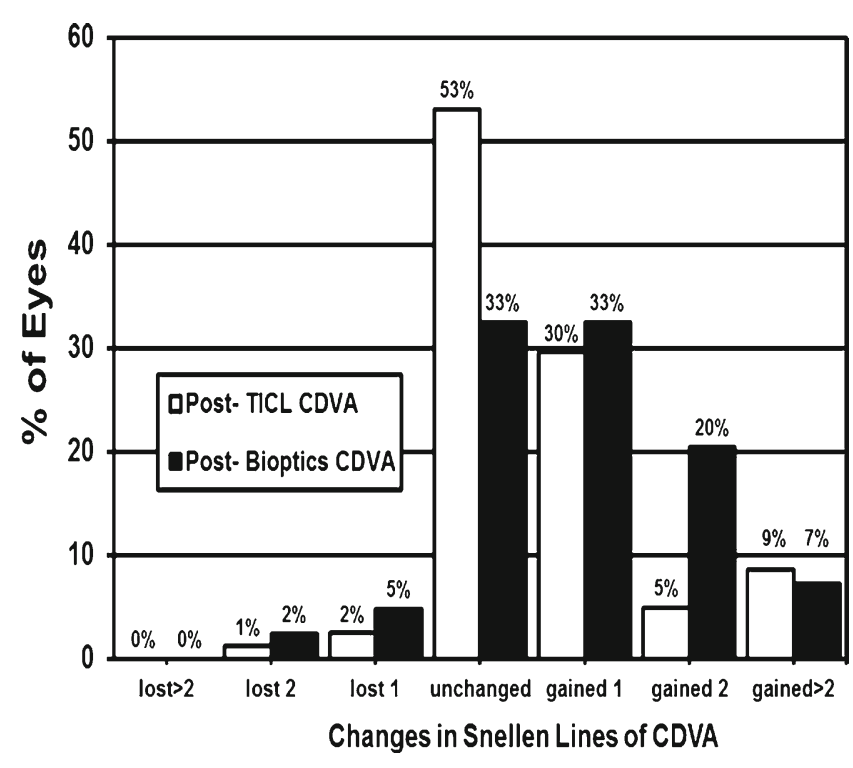

Fig. 7 Changes in CDVA (safety) in the TICL and bioptics groups

Two eyes in the TICL group were re-treated to correct TICL decentration.

\section{Discussion}

Some studies have reported visual and refractive outcomes after bioptics [8, 9, 11, 12] and TICL implantation [13-16] and have shown those procedures as effective and safe to correct moderate to high myopic astigmatism. However, there are few studies reporting whether TICL implantation is as effective as bioptics [17].

In the present study, postoperative UDVA and CDVA were similar between TICL and bioptics, although only CDVA was statistically better in the TICL group ( $p=$ 0.016). TICL implantation was comparable to bioptics in terms of safety and efficacy, although efficacy index was significantly higher in the bioptics group $(0.98 \pm 0.20$ vs. $1.09 \pm 0.19$ for TICL and bioptics, respectively, $p<0.001)$. These slightly better results for efficacy index in bioptics may be explained by the small residual uncorrected astigmatism of about 1.0 D that was observed in the TICL group. This may be due to a slight misalignment of the TICL, particularly in eyes with high refractive cylinder in which a slight misalignment may significantly worsen UDVA. With regard to predictability, TICL implantation and bioptics showed excellent and comparable results, although only J0 astigmatic component was significantly better in the bioptics group $(p=0.030)$. Postoperative spherical equivalent was reduced from a mean $-6.62 \pm 2.83 \mathrm{D}$ to $-0.15 \pm$ $0.36 \mathrm{D}$ for TICL and $-10.61 \pm 3.27 \mathrm{D}$ to $-0.08 \pm 0.26 \mathrm{D}$ in bioptics, with $81.5 \%$ and $94.0 \%$ of eyes within $\pm 0.50 \mathrm{D}$ of the predicted refraction in TICL and bioptics, respectively.
Frequently, the treatment of high myopia with astigmatism needs the combined use of different surgical procedures to achieve optimal refractive results. Concepts such as bioptics and adjustable refractive surgery refer to this combination. In 1999, Zaldivar et al. [8] used LASIK to correct the residual refractive error after ICL implantation for high myopia. They reported a mean postoperative spherical equivalent and refractive cylinder of $-0.20 \pm 0.90 \mathrm{D}$ and $-0.50 \pm$ $0.50 \mathrm{D}$, respectively, with $67 \%$ of eyes within $\pm 0.50 \mathrm{D}$ of emmetropia. Sánchez-Galeana et al. [9] successfully performed LASIK or PRK in 37 eyes implanted with an ICL for high myopia and final refraction was within $\pm 0.50 \mathrm{D}$ in $83.7 \%$ of eyes. In another study, Arne et al. [10] reported an improvement in UDVA in all eyes after bioptics; however, a loss of one line of CDVA occurred in $22.2 \%$ of PRK-treated eyes and in $13.6 \%$ of LASIK-treated eyes after ICL implantation. In the present study, bioptics showed similar visual and refractive results when compared to TICL implantation, however, bioptics requires time due to the interval between the two surgical procedures (mean 4.13 months in our study) and the patients' eyes are exposed to the inherent risks of a double surgical procedure.

As mentioned above, in recent years, toric ICLs have been shown to be effective for the correction of high myopic astigmatism. In the FDA toric ICL clinical study [13], the majority of the patients had postoperative UCVA better than or equal to their preoperative CDVA with more than $95 \%$ of eyes within $\pm 1.0 \mathrm{D}$ of intended refraction; adverse advents such as TICL rotation and clinically significant lens opacity occurred in a small percentage of eyes and were successfully treated with no loss in CDVA. Better refractive results were obtained in the present study for TICL group, although the mean preoperative astigmatism was significantly higher than in the FDA toric ICL cohort $(3.09 \pm 1.18 \mathrm{D}$ vs. $1.93 \pm$ 0.84). When compared with laser refractive treatments, toric ICLs performed better than PRK [22], LASIK [23], or wavefront-guided LASIK [24], besides, a toric ICL can be easily and safely repositioned even if improper alignment of the axis happens, as occurred with two toric ICLs in the present study.

Choi et al. [17] recently compared the clinical results between TICL implantation and bioptics to correct myopia and astigmatism and reported better visual outcomes for the TICL group with a large proportion of eyes gained better postoperative UDVA than preoperative CDVA. In addition, they reported a higher proportion of SE change $>0.50 \mathrm{D}$ from 1 to 12 months in the bioptics procedure. In the present study, we found similar results regarding visual acuity; however, our results seem to be better regarding refractive outcomes. Although we have not analyzed stability of the refraction over different months, it will probably have similar behavior once bioptics is likely to involve greater and longer corneal wound healing. 
A limitation of the present study is that both groups were very different regarding preoperative characteristics of age, gender, refractive sphere, and refractive cylinder, and should be taken into account in the interpretation of the present results. In fact, the higher number of eyes gaining lines of visual acuity observed in the bioptics group may be explained by the a priori higher myopia in this group, which in turn may reflect the result of magnification of the retinal image by eliminating the spectacle-induced minification that those patients experience preoperatively. However, astigmatism correction was the main issue with both procedures and, despite the differences in preoperative refractive cylinder magnitude, we do not observed significant differences for postoperative J0 and J45 astigmatic components, which better reflects the statistical comparability between both groups preoperatively.

Increased intraocular pressure, pupillary block, and cataract formation, have been the most documented safety concerns related to ICL implantation [25-27]. Frequently it has been associated with the physical interaction between the ICL and crystalline lens or with the iris. Sánchez-Galeana [9] reported the development of anterior subcapsular opacities in three eyes several weeks after excimer laser surgery in eyes containing ICL for high myopia. They also reported ocular hypertension and macular hemorrhage in one eye each. Choi et al. [17] reported two crystalline lens opacities after bioptics and they assume to be due to low vaulting of the implanted ICL. In the present study, there were no cases of chronic elevated postoperative IOP or cataract development, but the relatively short follow-up should be considered as a limitation. In fact, the duration of follow-up should be taken into consideration given that the occurrence of cataract is higher in patients with longer follow-up [25, 27]. Furthermore, it has been reported that vaulting has a tendency to decrease over time leading to an increased risk of cataract formation, while further mechanical contact between the ICL and the iris was considered as the most important cause of later increased IOP events. Another limitation of the present study is that we do not evaluate the exact position of the ICL in the sulcus and, by this, we cannot accurately predict if there was a slight misalignment in the TICL or a slight rotation of the TICL overtime that may explain some less good results in the TICL group.

The goal of refractive surgery is to achieve emmetropia through any corrective procedure and therefore the existence of toric IOLs became essential. Both TICL implantation and bioptics showed good clinical results in patients with myopic astigmatism, reducing preoperative spherical and astigmatic errors with high predictability and safety. Despite that the bioptics procedures showed slightly better outcomes in some clinical measures such as uncorrected visual acuity, efficacy, and refractive predictability, TICL implantation may result in a more effective procedure since it avoids subsequent laser ablation and therefore eliminates the risks of a second procedure or changes in the optical quality of the cornea through laser ablation.

Acknowledgments and disclosure The authors have no proprietary interests in any of the materials mentioned in this article. This research was supported in part by a Universitat de Valencia Research Grant to Robert Montés-Micó (\#SAF2009-13342 and \#SAF2008-01114-E\#) and Fundação para a Ciência e Tecnologia of Portugal through a Grant to Paulo Fernandes (\#FCT-SFRH-BD-34303-2007\#).

\section{References}

1. American Academy of Ophthalmology (1999) Excimer laser photorefractive keratectomy (PRK) for myopia and astigmatism. Ophthalmology 106:422-437

2. Sugar A, Rapuano CJ, Culbertson WW, Huang D, Varley GA, Agapitos PJ, de Luise VP, Koch DD (2002) Laser in situ keratomileusis for myopia and astigmatism: safety and efficacy: a report by the American Academy of Ophthalmology. Ophthalmology 109:175-187

3. Schallhorn SC, Farjo AA, Huang D, Boxer Wachler BS, Trattler WB, Tanzer DJ, Majmudar PA, Sugar A (2008) Wavefront-guided LASIK for the correction of primary myopia and astigmatism a report by the American Academy of Ophthalmology. Ophthalmology 115:1249-1261

4. Kamiya K, Shimizu K, Igarashi A, Hikita F, Komatsu M (2009) Four-year follow-up of posterior chamber phakic intraocular lens implantation for moderate to high myopia. Arch Ophthalmol 127:845-850

5. Sanders DR, Doney K, Poco M (2004) United States Food and Drug Administration clinical trial of the Implantable Collamer Lens (ICL) for moderate to high myopia: three-year follow-up. Ophthalmology 111:1683-1692

6. Alfonso JF, Baamonde B, Fernandez-Vega L, Fernandes P, Gonzalez-Meijome JM, Montes-Mico R (2011) Posterior chamber collagen copolymer phakic intraocular lenses to correct myopia: five-year follow-up. J Cataract Refract Surg 37:873-880

7. Uusitalo RJ, Aine E, Sen NH, Laatikainen L (2002) Implantable contact lens for high myopia. J Cataract Refract Surg 28:29-36

8. Zaldivar R, Davidorf JM, Oscherow S, Ricur G, Piezzi V (1999) Combined posterior chamber phakic intraocular lens and laser in situ keratomileusis: bioptics for extreme myopia. J Refract Surg 15:299-308

9. Sanchez-Galeana CA, Smith RJ, Rodriguez X, Montes M, Chayet AS (2001) Laser in situ keratomileusis and photorefractive keratectomy for residual refractive error after phakic intraocular lens implantation. J Refract Surg 17:299-304

10. Arne JL, Lesueur LC, Hulin HH (2003) Photorefractive keratectomy or laser in situ keratomileusis for residual refractive error after phakic intraocular lens implantation. J Cataract Refract Surg 29:1167-1173

11. Guell JL, Vazquez M, Gris O (2001) Adjustable refractive surgery: 6-mm Artisan lens plus laser in situ keratomileusis for the correction of high myopia. Ophthalmology 108:945-952

12. Munoz G, Alio JL, Montes-Mico R, Belda JI (2003) Anglesupported phakic intraocular lenses followed by laser-assisted in situ keratomileusis for the correction of high myopia. Am J Ophthalmol 136:490-499

13. Sanders DR, Schneider D, Martin R, Brown D, Dulaney D, Vukich J, Slade S, Schallhorn S (2007) Toric Implantable Collamer Lens for moderate to high myopic astigmatism. Ophthalmology 114:5461 
14. Alfonso JF, Baamonde B, Madrid-Costa D, Fernandes P, Jorge J, Montes-Mico R (2010) Collagen copolymer toric posterior chamber phakic intraocular lenses to correct high myopic astigmatism. J Cataract Refract Surg 36:1349-1357

15. Alfonso JF, Fernandez-Vega L, Fernandes P, Gonzalez-Meijome JM, Montes-Mico R (2010) Collagen copolymer toric posterior chamber phakic intraocular lens for myopic astigmatism: one-year follow-up. J Cataract Refract Surg 36:568-576

16. Kamiya K, Shimizu K, Aizawa D, Igarashi A, Komatsu M, Nakamura A (2010) One-year follow-up of posterior chamber toric phakic intraocular lens implantation for moderate to high myopic astigmatism. Ophthalmology 117:2287-2294

17. Choi SH, Lee MO, Chung ES, Chung TY (2011) Comparison of the Toric Implantable Collamer Lens and bioptics for myopic astigmatism. J Refract Surg 27:91-97

18. Alfonso JF, Lisa C, Palacios A, Fernandes P, Gonzalez-Meijome JM, Montes-Mico R (2009) Objective vs. subjective vault measurement after myopic Toric Implantable Collamer Lens implantation. Am J Ophthalmol 147:978-983

19. Alfonso JF, Lisa C, Abdelhamid A, Fernandes P, Jorge J, MontesMico R (2010) Three-year follow-up of subjective vault following myopic Toric Implantable Collamer Lens implantation. Graefes Arch Clin Exp Ophthalmol 248:1827-1835

20. Alfonso JF, Fernandez-Vega L, Lisa C, Fernandes P, GonzalezMeijome JM, Montes-Mico R (2010) Collagen copolymer toric posterior chamber phakic intraocular lens in eyes with keratoconus. J Cataract Refract Surg 36:906-916

21. Thibos LN, Horner D (2001) Power vector analysis of the optical outcome of refractive surgery. J Cataract Refract Surg 27:80-85

22. Schallhorn S, Tanzer D, Sanders DR, Sanders ML (2007) Randomized prospective comparison of Visian Toric Implantable Collamer Lens and conventional photorefractive keratectomy for moderate to high myopic astigmatism. J Refract Surg 23:853-867

23. Sanders DR, Sanders ML (2008) Comparison of the Toric Implantable Collamer Lens and custom ablation LASIK for myopic astigmatism. J Refract Surg 24:773-778

24. Kamiya K, Shimizu K, Igarashi A, Komatsu M (2008) Comparison of Collamer toric contact lens implantation and wavefrontguided laser in situ keratomileusis for high myopic astigmatism. J Cataract Refract Surg 34:1687-1693

25. Fernandes P, Gonzalez-Meijome JM, Madrid-Costa D, FerrerBlasco T, Jorge J, Montes-Mico R (2011) Implantable Collamer posterior chamber intraocular lenses: a review of potential complications. J Refract Surg 27:765-776

26. Sanders DR (2008) Anterior subcapsular opacities and cataracts 5 years after surgery in the Visian Toric Implantable Collamer Lens FDA trial. J Refract Surg 24:566-570

27. Kohnen T, Kook D, Morral M, Guell JL (2010) Phakic intraocular lenses: part 2: results and complications. J Cataract Refract Surg $36: 2168-2194$ 